\title{
Sensing (un)binding events via surface plasmons: Effects of resonator geometry
}

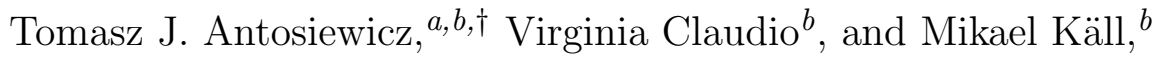 \\ ${ }^{a}$ Centre of New Technologies, University of Warsaw, Banacha 2c, 02-097 Warsaw, Poland; \\ ${ }^{b}$ Department of Physics, Chalmers University of Technology, \\ SE-412 96 Göteborg, Sweden
}

\begin{abstract}
The resonance conditions of localized surface plasmon resonances (LSPRs) can be perturbed in any number ways making plasmon nanoresonators viable tools in detection of e.g. phase changes, $\mathrm{pH}$, gasses, and single molecules. Precise measurement via LSPR of molecular concentrations hinge on the ability to confidently count the number of molecules attached to a metal resonator and ideally to track binding and unbinding events in real-time. These two requirements make it necessary to rigorously quantify relations between the number of bound molecules and response of plasmonic sensors. This endeavor is hindered on the one hand by a spatially varying response of a given plasmonic nanosensor. On the other hand movement of molecules is determined by stochastic effects (Brownian motion) as well as deterministic flow, if present, in microfluidic channels. The combination of molecular dynamics and the electromagnetic response of the LSPR yield an uncertainty which is little understood and whose effect is often disregarded in quantitative sensing experiments. Using a combination of electromagnetic finite-difference time-domain (FDTD) calculations of the plasmon resonance peak shift of various metal nanosensors (disk, cone, rod, dimer) and stochastic diffusion-reaction simulations of biomolecular interactions on a sensor surface we clarify the interplay between position dependent binding probability and inhomogeneous sensitivity distribution. We show, how the statistical characteristics of the total signal upon molecular binding are determined. The proposed methodology is, in general, applicable to any sensor and any transduction mechanism, although the specifics of implementation will vary depending on circumstances. In this work we focus on elucidating how the interplay between electromagnetic and stochastic effects impacts the feasibility of employing particular shapes of plasmonic sensors for real-time monitoring of individual binding reactions or sensing low concentrations - which characteristics make a given sensor optimal for a given task. We also address the issue of how particular illumination conditions affect the level of uncertainty of the measured signal upon molecular binding.
\end{abstract}

Keywords: plasmonic sensors, diffusion-reaction simulations, signal uncertainty, single-molecule sensing, shape effect

\section{INTRODUCTION}

An important challenge to be overcome in life sciences are the limitations associated with ensemble-level measurements, as ensemble-level methodology may obscure molecular properties due to averaging and as a consequence make it challenging to identify reaction dynamics and concentration values. While considerable information might be obtained, ${ }^{1,2}$ these problems become apparent in cases when molecular concentration is low and the measurement technique offers low sensitivity. Improvements in such sensing experiments are, of course, introduced by improving the sensitivity of the transducting mechanism which converts changes in the sensor's environment into a measurable signal of high fidelity. This last requirement is of vital importance regardless of the physical mechanism used to probe a target analyte. To obtain a signal from molecules whose dimensions may be as small as a few nanometers special techniques are necessary. Commonly used are fluorescence microscopies in which a light emitting label is chemically bound to the target and emitted photons indicate the presence of the analyte,,$^{3-5}$ however, labels may affect the kinetic behavior of molecules. Forgoing fluorescent labeling is possible as recent demonstrations of direct single-molecule optical absorption

\footnotetext{
${ }^{\dagger}$ tomasz.antosiewicz@uw.edu.pl
}

Nanophotonics VI, edited by David L. Andrews, Jean-Michel Nunzi, Andreas Ostendorf, Proc. of SPIE Vol. 9884, 98842F · C 2016 SPIE · CCC code: 0277-786X/16/\$18 · doi: 10.1117/12.2227119 
has been demonstrated, ${ }^{6,7}$ yet in this method extremely low noise levels are required for detecting optical absorption.

The above mentioned are not the only examples of methods of detecting single molecules. A very vibrant field is one which employs coherent oscillations of conduction electrons and electromagnetic fiels at a metaldielectric interface. ${ }^{8}$ These so called surface plasmon-polaritons are characterized by strong amplifications of the electric field near the metal surface, in essence amplifying the electromagnetic interaction between the metal supporting the plasmon and an object placed in the enhanced field. ${ }^{9}$ This and other properties of plasmons have been behind the incorporation of metal surfaces and nanoparticles in a myriad of application from biosensing, ${ }^{10,11}$ photodetection, ${ }^{12,13}$ and waveguiding, ${ }^{14,15}$ to light emission, ${ }^{16,17}$ plexcitonics ${ }^{18,19}$ and materials science. ${ }^{20,21}$ When utilized as a sensor, the plasmon resonance serves as a probe of the environment surrounding the metal nanoparticle and, being sensitive to the spatial distribution of the refractive index (permittivity), responds with a shift of the peak position when the distribution changes. ${ }^{9,22,23}$ In a general case of permittivity changes in the $\epsilon>1$ range of the surrounding, an increase of $\epsilon$ results in a redshift of the resonance wavelength and a blue shift in the opposite case. When the sensed material is also a metal ${ }^{24}$ or a dielectric with a large refractive index (so that is can support resonances of it $\mathrm{own}^{25}$ ), then coupling between the resonance of the metan nanosensor and the sensed object is more complex and one cannot a priori determine the resuling resonance shift. ${ }^{24}$ However, in the case of proteins, whose typical refractive indices are approximately $10 \%$ larger than of water ${ }^{26}$ colorimetric localized surface plasmon resonance (LSPR) sensing is an appropriate tool for studying their association and dissociation. An important point here is that to assure sensor specificity, the sensor surface is functionalized with receptors that target a specific analyte. ${ }^{27,28}$

As we have noted in the beginning, it is not always enough to measure the ensemble-averaged response of a large number of nanoparticle sensors, even though the sensitivity can be on the order of (or leven lower than) one molecule per nanoparticle. ${ }^{29}$ The defficiency becomes apparent when one strives to establish a clear and very accurate relationship between a measured peak shift $(\Delta \lambda)$ and the currently bound number of molecules $N$. I.e. - how does one design an accurate LSPR molecule counter? With the recent demonstration of single molecule detection with an LSPR sensor ${ }^{30}$ and real-time detection of individual binding events to a nanosensor ${ }^{31}$ basic proof of concept has been shown. However, to extend these accomplishments to quantitative use in molecule counting and determining binding rates it is crucial to know the confidence level offered by specific nanosensors. Moreover, this confidence level will, in all probability, be also affected by the experimental setup, data analysis, and the sampled object. To quantify this accuracy one must account for the nonuniform LSPR signal as well as the stochastic nature of diffusion and binding reactions that bring the analyte to the sensor. The inhomogeneity of the LSPR near-field is of prime concern. For example, nanocones may exhibit a two order of magnitude variability of the local resonance peak shift; similar observations may be made for nanoparticle dimers ${ }^{32}$ or other more complex shapes. ${ }^{33,34}$ Thus, any conversion of an arbitrary peak shift to the number of molecules causing it will exhibit an uncertainty. Furthermore, the stochastic nature of molecular movement and the shape of a particular resonator will result in nonuniform binding probablilities. One also has to take into account signal noise and drift. Hence, the accuracy of counting molecules (and as consequence measuring concentration) poses a nontrivial challenge. Finally, we note that the above issues will also apply to measuring binding rate constants and molecular affinities from binding time traces, ${ }^{35}$ however, their accuracy is expected to depend on the inhomogeneity of the LSPR.

To address these issues and ascertain their influence on LSPR single- and few-molecule sensing we design a multiscale approach in which we combine the electromagnetic properties of metal nanoresonators with stochastic diffusion-reaction modelling. ${ }^{32}$ Using numerical calculations we obtain peak shift (sensitivity) maps. Brownian diffusion and probabilistic reactions simulate molecular trajectories around a nanosensor. When both are combined we obtain a temporal peak shift trace that mimics real sensing experiments. We analyse these time traces as well as the constituent elements to quantify the sensing uncertainty introduced by various types of plasmonic sensors.

\section{METHODS}

There are two parts to the in silico experiments we carry out. Due to the different nature of the issues each consideres - electromagnetic modelling and stochastic diffusion-reaction simulations - we carry out the 

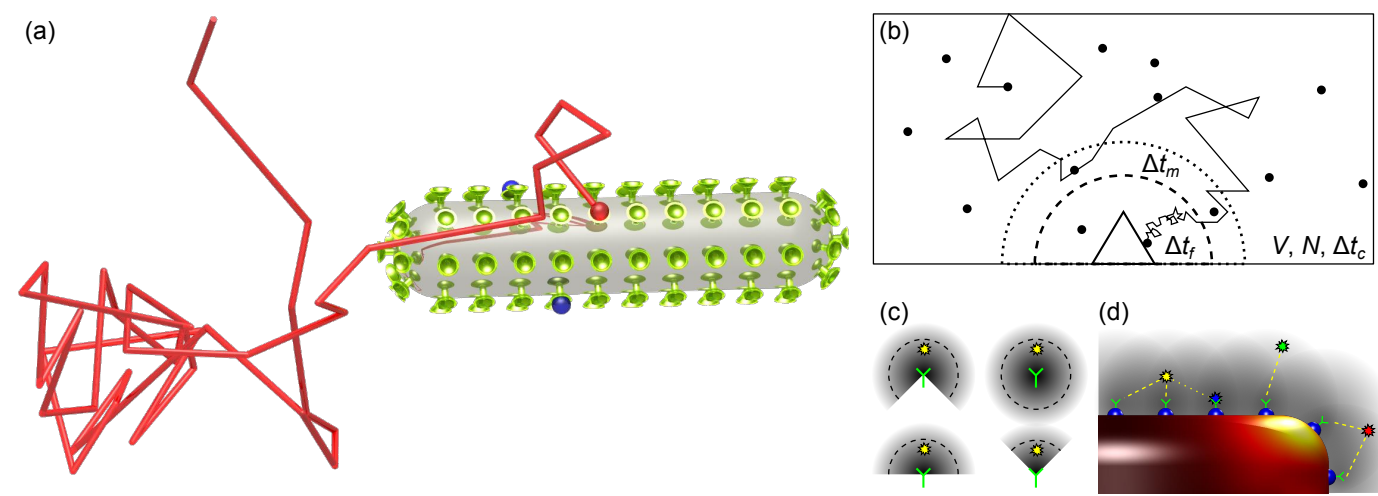

(c)

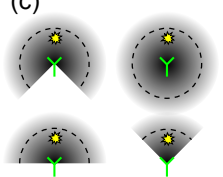

(d)

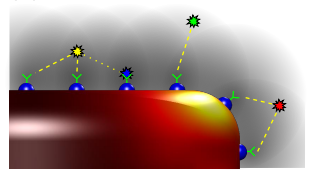

(e)
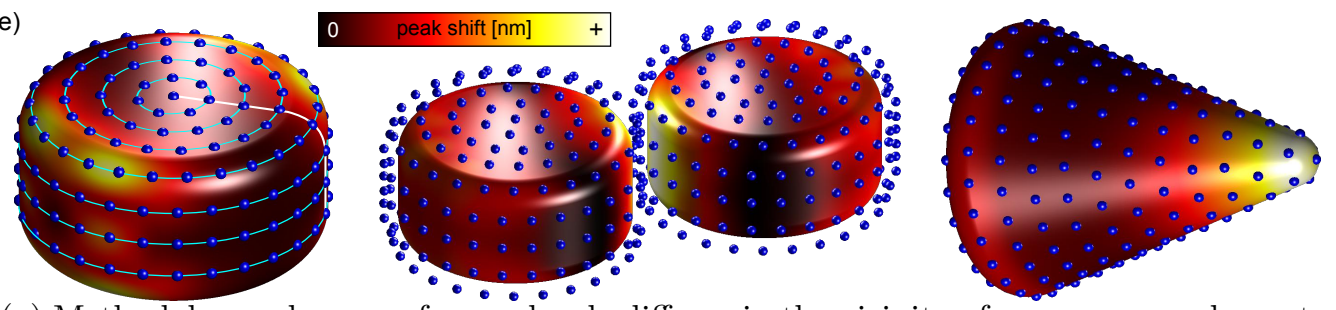

Figure 1. (a) Methodology scheme: a free molecule diffuses in the vicinity of a nanosensor decorated by receptors. In each time step $\Delta t$ is traverses a stochastic distance and at each time step a binding probability to nearby receptors (if present) is calculated. The molecules do not interact with one another during diffusion, but cannot bind to previously occupied receptors. (b) $N$ diffusing molecules are contained in a box of volume $V$ with a receptor placed on a substrate (one side of the box). Three zones with different time steps are defined to achieve a ballance between speed, code complexity, and accuracy of molecules diffusing very close to the sensor. (c) Illustarion of the reaction volume and the resulting volume exclusion effect. (d) The binding probability of a molecule at each time step depends on the number of free receptors in its vicinity. (e) Spatial 3D maps of peak shift sensitivity with indicated receptor positions at the surface of considered resonators.

calculation separately. The electromagnetic part is done by finite-difference time-domain (FDTD) calculations (FDTD Solutions 8.7.1, Lumerical, Inc.), while the Brownian dynamics part is simulated in an in-house $\mathrm{C}++$ code. The details are given in the following sections. ${ }^{32}$

\subsection{Electromagnetic calculations}

In order to keep the in silico experiments simple we consider a predetermined mesh of receptors spaced in such a way that allows for molecules to be bound to all receptors without spatial overlap. The molecule is assumed to be a $6 \mathrm{~nm}$ dielectric sphere $(n=1.5)$. For each nanosensor we calculate a semi-regular mesh of ca. equidistant points at which we sequentially place the molecule. For each distinct position occupied by a molecule (sphere) we calculate the extinction spectrum and compare it to one of a clean nanosensor. The resonators are assumed to be made of silver with slightly increases losses with permittivity described by the Drude function $\epsilon(\omega)=\epsilon_{\infty}-\omega_{p}^{2} /(\omega(\omega+i \gamma))$, where the parameters are $\epsilon_{\infty}=3.7, \hbar \omega_{p}=8.55 \mathrm{eV}$, and $\hbar \gamma=130 \mathrm{meV}$. The spatial discretization is $0.5 \mathrm{~nm}$, we use the Total-Field/Scattered-Field formalism to introduce the incident linearly polarized wave and scattered energy is absorbed by the boundary conditions. Figure 1e shows some of the considered nanosensor geometries with their surfaces decorated by the 3D spatial sensitivity maps.

\subsection{Diffusion-reaction simulations}

Figure 1a,b shows a scheme of our simulations. Molecules diffuse in a cubic volume $V$ which contains the nanosensor places at one of the sides. The molecules do not interact with one another except through the occupancy state of the receptors (Fig. 1c,d. Diffusion is assumed to follow a Wiener process in which the mean one-dimensional diffusion length $s=\sqrt{2 D \Delta t}$ with $D$ being the diffusion constant. Diffusion steps 

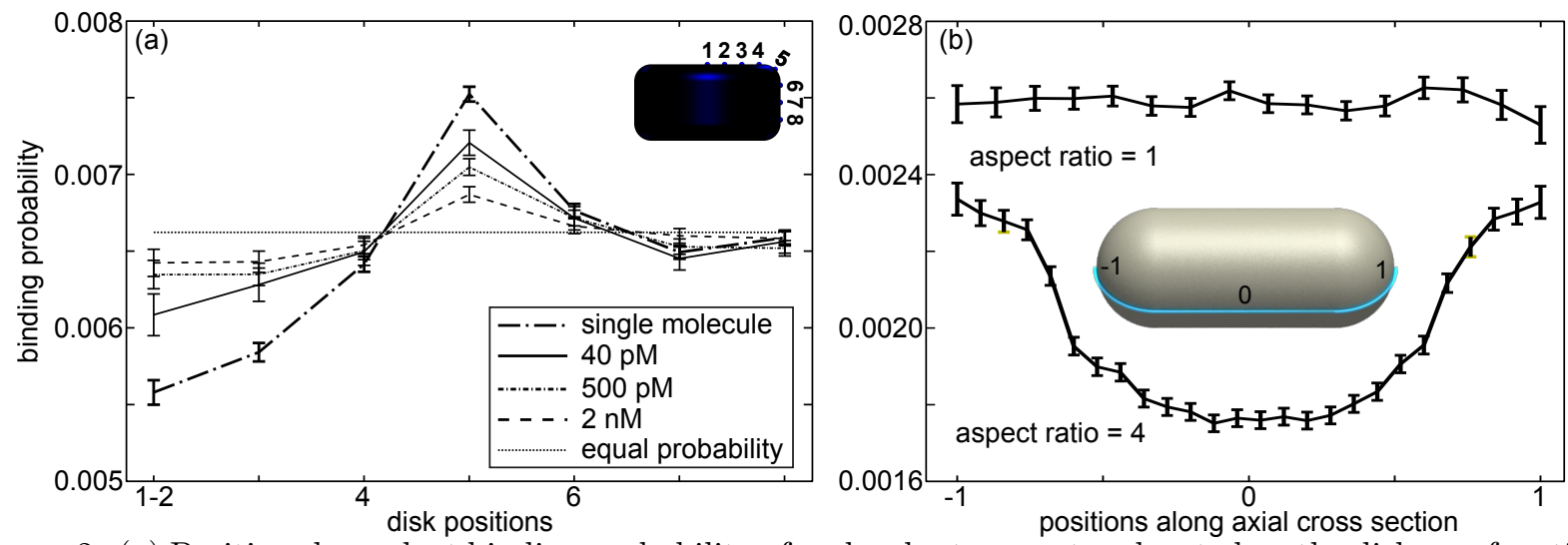

Figure 2. (a) Position-dependent binding probability of molecules to receptors located on the disk as a function of molecular concentration. For single molecule considerations there is considerable variation between the probabilities reaching ca. 40\%. As the concentration increases more receptors become occupied and the binding probability flattens and ultimately for very large concentrations approaches a uniform value. (b) Single molecule binding probability for two rods (in solution, not on a substrate) of aspect rations 1 (sphere) and 4. In the first case the binding probability is flat, as it should be, while for the rod with the aspect ratio of 4 the probability of a molecule to bind at the ends is $33 \%$ higher than in the middle.

are calculated by drawing random numbers from the normal $(0,1)$ distribution and multiplying it by $s$. To accurately model diffusion around the complex-shaped nanosensor and at the same time use an efficient time step for molecules far away from the sensor, we employ three time steps. The shortest time step is used for molecules close to the sensor $\left(\Delta t_{f}=0.1 \mu \mathrm{s}\right)$, while further away they increase 100- and 10000-fold. These time steps were determined through convergence testing and give good accuracy. ${ }^{32}$ In each time step we also check that molecules have not entered or passed through the sensor.

When a diffusing molecule is in range of at least one receptor we calculate its binding probability accorting to $P_{b}=1-\exp \left(-\Sigma\left(\Delta t_{f} k_{o n} / V_{r}\right)\right)$, where $V_{r}$ is the spherical reaction volume given by the distance between the molecule and the receptor whose binding probability is evaluated, $k_{o} n$ is the binding reaction rate and the sum runs over all receptors in range of the diffusing molecule. Naturally, only free receptros are considered. A random number $r_{1}$ in the $0-1$ interval is drawn (uniformly distributed) and if $r_{1}<P_{b}$ then binding occurrs. If more than one receptor contributes to the sum for $P_{b}$, a second random number $r_{2}$ is used to evaluate which receptor binds the molecule. This is done by comparing $r_{2}$ with the cumulative sum of individual binding probabilities to eligible receptors. Once bound, at each time step the unbinding probability is compared to another random number to see if bound molecules resume diffusion. We used $k_{\text {on }}=10^{6} \mathrm{M}^{-1} \mathrm{~s}^{-1}$ and the unbinding rate was $k_{\text {off }}=0.005 \mathrm{~s}^{-1}$, based on commonly considered reaction kinetics. ${ }^{36}$ We considered a span of concentrations from a few tens of $\mathrm{pM}$ to a few $\mathrm{nM}$, in each case this was determined by the size of the diffusion box volume $V$ and number is molecules $N$.

\section{RESULTS AND DISCUSSION}

In our analysis we first focus on the stochastic aspects - Figure 2 shows how the binding probability depends on the position of the receptor on the surface of a nanodisk (Fig. 2a) and a nanorod (Fig 2b). In the first case we compare the single molecule binding probability (alternatively the first molecule probability) with probabilities for increasing concentration. In the second we compare the binding probability of a nanosphere to a rod (aspect ratios of 1 vs. 4). When there are only small numbers of bound molecules the location of arriving molecules depends on the shape. This means that in the beginning one is more likely to observe peak shifts from those areas - and if the structure is engineered properly, those peak shifts are large. This is of great benefit for yes/no types of sensors. However, it is also necessary to keep in mind that those areas with preferential binding may harbor only small numbers of receptors what will lower this method of nanosensor optimization. 

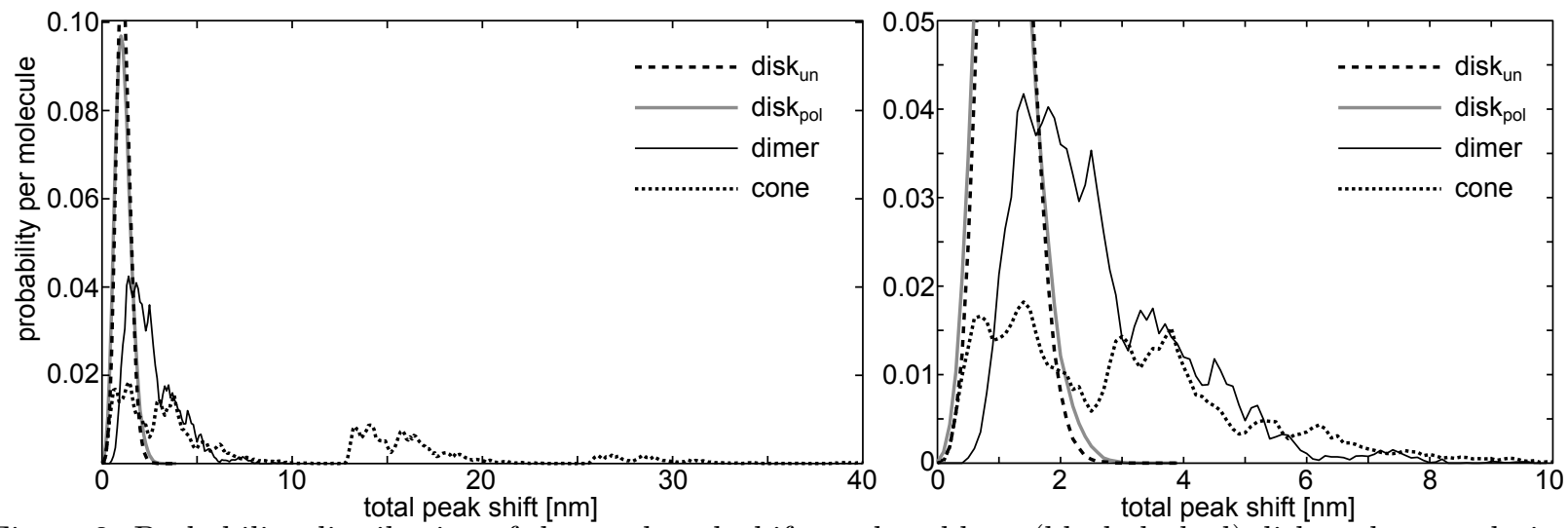

Figure 3. Probability distribution of the total peak shift produced by a (black dashed) disk under unpolarized illumination, (solid gray) disk under linerar polarization, (thin black) dimer, and (black dotted) cone for a molecular concentration of $90 \mathrm{pM}$; the probability is normalized to the average number of molecules bound to each sensor. The disks under both types of illumination give a similar, very narrow distribution making this sensor the most accurate in determining the number of bound molecules even though the maximum peak shifts are much smaller than for the dimer and cone. The last two present peak shift distributions which are much broader and in the case of the cone, have a repeating quasi-periodic nature. This broadness makes the accuracy of counting molecules bound to the sensor much worse than for the disk.

The second issue is with the evolution of the binding probability with concentration. The single molecule case for the nanodisk corresponds to a $0.66 \%$ coverage (151 receptors), while the $40 \mathrm{pM}$ to a $6 \%$ coverage (9 bound molecules). From Fig. 2a it can be seen, that this first step to $40 \mathrm{pM}$ makes the biggest difference on the binding probability ( $10 \%$ change in the center). Further increases to even $2 \mathrm{nM}$ (50-fold) change it only by $5 \%$ more and the binding probability is at this point almost uniform. Such effects are even more pronounced for nanocone sensors. ${ }^{32}$

The relationship between the location of binding molecules and concentration/coverage becomes even more important when the inhomogeneous peak shifts of the nanoresonators are considered. We plot the results of combined electromagnetic and stochastic calculations in which we convert the number-of-bound-molecules time traces from the latter to peak shift time traces. We then analyse these traces and calculate the probabilities of measuring particular peak shifts. For the disk we also add unpolarized illumination conditions. These results are shown in Fig. 3 (the right plot is a magnification of the left part of the left plot). The first two cases - disks under linearly polarized (black dashed) and unpolarized (thick gray) illuminarion show the narrowest distribution with maxima around $1.1 \mathrm{~nm}$ and standard deviations on the order of 0.3 . This gives much better accuracy in counting molecules than the remaining sensors - the dimer and cone. These last two exhibit extremely broad distributions with the cone displaying a repeating, quasi-periodic pattern. Such broad distributions make the exact number of bound molecules subject to greater uncertainty, although these will feature bettern yes/no properties than the disks. Of particular interest is the cone, whose repeating pattern is rather curious. It is the result of having relatively few high sensitivity sites which produce a very large peak shift and each "discrete" pattern is the result of having $0,1,2$, etc. molecules bound at that the apex of the cone. With ca. 15 molecules bound to the cone this means that this single event (if we consider only the first two patterns) can result in a peakshift larger than the remaining 14 molecules, what confirms the poor suitability of the cone as a quantitative sensor. However, as a qualitative yes/no type of sensor it is excellent.

\section{CONCLUSIONS AND SUMMARY}

Our results show that interpreting signals obtained by a plasmonic sensor of complex geometry and consequently complex spatial response is not trivial. Indeed, the relationship between plasmonic resonators and 
molecule localization has been receiving increasing interest in recent years. These addressed the inhomogeneity of the LSPR response to optimize a disk- ${ }^{37}$ or rod-shaped ${ }^{38}$ sensor, although without explicitly taking into account the stochastic nature of binding for a disk or employing a quasi-static continuum model to describe interactions between plasmons and non-absorbing molecules. ${ }^{39}$ Here, we show that even for very simple sensors - e.g. a disk - it is not possible to have always accuracy on the order of a few pecent - in many cases it may be on the order of the measured (mean) value. This estimation does not even have to take into account the Poissonian behavior of molecule binding and variance of number of bound molecules. Already the inhomogeneous sensor response is enough to cause considerable spread of the signal, while the Poissonian statistics make the accuracy worse. However, at a certain point for enough bound molecules the stochastic nature of such experiments may help reduce the uncertainty due to the fact that the relative accuracy proportional to $1 / \sqrt{N}$ will be the dominating effect. While here we have only discussed the impact of sensor inhomogeneity on counting the number of bound molecules and its accuracy, the consequences of a nonuniform response extend to measuring molecular concentration, which is directly linked to the sensor coverage, and analysing fluctuations of the temporal signals for reaction rate extraction. ${ }^{40,41}$

In summary, we have decribed a method to reproduce in silico a common, yet complex sensing experiment utilizing the peak shift of a plasmonic nanoantenna as a transducting mechanism. This approach allows one to analyse all the elements of a sensing experiment individually, understand how they interact with one another and utilizing this knowledge design a plasmonic sensor that is optimal for any given application, whether it is to report on the presence (or lack thereof) of an analyte or to measure binding kinetics and extract rates.

\section{REFERENCES}

[1] Deniz, A. A., Mukhopadhyay, S., and Lemke, E. A., "Single-molecule biophysics: At the interface of biology, physics and chemistry," J. R. Soc. Interface 5, 15-45 (2008).

[2] Coelho, M., Maghelli, N., and Tolic-Norrelykke, I. M., "Single-molecule imaging in vivo: The dancing building blocks of the cell," Integr. Biol. 5, 748-758 (2013).

[3] Denk, W., Strickler, J., and Webb, W., "Two-photon laser scanning fluorescence microscopy," Science 248, 73-76 (1990).

[4] Sánchez, E., Novotny, L., and Xie, X., "Near-field fluorescence microscopy based on two-photon excitation with metal tips," Phys. Rev. Lett. 82, 4014-4017 (1999).

[5] Hell, S. W., "Far-field optical nanoscopy," Science 316, 1153-1158 (2007).

[6] Gaiduk, A., Yorulmaz, M., Ruijgrok, P. V., and Orrit, M., "Room-temperature detection of a single molecule's absorption by photothermal contrast," Science 330, 353-356 (2010).

[7] Chong, S., Min, W., and Xie, X. S., "Ground-state depletion microscopy: Detection sensitivity of singlemolecule optical absorption at room temperature," J. Phys. Chem. Lett. 1, 3316-3322 (2010).

[8] Maier, S., [Plasmonics: Fundamentals and Applications], Springer-Verlag (2007).

[9] Antosiewicz, T. J., Apell, S. P., Claudio, V., and Käll, M., "A simple model for the resonance shift of localized plasmons due to dielectric particle adhesion," Opt. Express 20, 524-533 (2012).

[10] Brolo, A. G., "Plasmonics for future biosensors," Nature Photon. 6, 709-713 (2012).

[11] Anker, J. N., Hall, W. P., Lyandres, O., Shah, N. C., Zhao, J., and Van Duyne, R. P., "Biosensing with plasmonic nanosensors," Nature Mater. 7, 442-453 (2008).

[12] Wu, W., Bonakdar, A., and Mohseni, H., "Plasmonic enhanced quantum well infrared photodetector with high detectivity," Appl. Phys. Lett. 96, 161107 (2010).

[13] Zheng, B. Y., Wang, Y., Nordlander, P., and Halas, N. J., "Color-selective and CMOS-compatible photodetection based on aluminum plasmonics," Adv. Mater. 26, 6318-6323 (2014).

[14] Saj, W. M., Antosiewicz, T. J., Pniewski, J., and Szoplik, T., "Energy transport in plasmon waveguides on chain of metal nanoplates," Opto-Electron. Rev. 14, 243-251 (2006).

[15] Ahmadivand, A. and Golmohammadi, S., "Compositional arrangement of rod/shell nanoparticles: an approach to provide efficient plasmon waveguides," Opto-Electron. Rev. 22(2), 101-108 (2014).

[16] Sun, G., Khurgin, J. B., and Soref, R. A., "Plasmonic light-emission enhancement with isolated metal nanoparticles and their coupled arrays," J. Opt. Soc. Am. B 25, 1748-1755 (Oct 2008). 
[17] Noginov, M. A., Zhu, G., Belgrave, A. M., Bakker, R., Shalaev, V. M., Narimanov, E. E., Stout, S., Herz, E., Suteewong, T., and Wiesner, U., "Demonstration of a spaser-based nanolaser," Nature 460, 1110-1113 (2009).

[18] Schlather, A. E., Large, N., Urban, A. S., Nordlander, P., and Halas, N. J., "Near-field mediated plexitonic coupling and giant Rabi splitting in individual metallic dimers," Nano Lett. 13, 3281-3286 (2013).

[19] Antosiewicz, T. J., Apell, S. P., and Shegai, T., "Plasmon-exciton interactions in a core-shell geometry: From enhanced absorption to strong coupling," ACS Photonics 1, 454-463 (2014).

[20] Ferrara, D. W., Nag, J., MacQuarrie, E. R., Kaye, A. B., and Haglund, R. F., "Plasmonic probe of the semiconductor to metal phase transition in Vanadium Dioxide," Nano Lett. 13, 4169-4175 (2013).

[21] Adibi, P. T. Z., Mazzotta, F., Antosiewicz, T. J., Skoglundh, M., Grönbeck, H., and Langhammer, C., "In situ plasmonic sensing of platinum model catalyst sintering on different oxide supports and in $\mathrm{O}_{2}$ and $\mathrm{NO}_{2}$ atmospheres with different concentrations," ACS Catal. 5, 426-432 (2015).

[22] Englebienne, P., "Use of colloidal gold surface plasmon resonance peak shift to infer affinity constants from the interactions between protein antigens and antibodies specific for single or multiple epitopes," Analyst 123, 1599-1603 (1998).

[23] Haes, A. J. and Van Duyne, R. P., "A nanoscale optical biosensor: Sensitivity and selectivity of an approach based on the localized surface plasmon resonance spectroscopy of triangular silver nanoparticles," J. Am. Chem. Soc. 124, 10596-10604 (2002).

[24] Syrenova, S., Wadell, C., Nugroho, F. A. A., Gschneidtner, T. A., Fernandez, Y. A. D., Nalin, G., Świtlik, D., Westerlund, F., Antosiewicz, T. J., Zhdanov, V. P., Moth-Poulsen, K., and Langhammer, C., "Hydride formation thermodynamics and hysteresis in indiviaual pd nanocrystals with different size and shape," Nature Mater. 14, 1236-1243 (2015).

[25] Schmidt, M. K., Esteban, R., Sáenz, J. J., Suárez-Lacalle, I., Mackowski, S., and Aizpurua, J., "Dielectric antennas - a suitable platform for controlling magnetic dipolar emission," Opt. Express 20, 13636-13650 (2012).

[26] Vörös, J., "The density and refractive index of adsorbing protein layers," Biophysical J. 87, 553-561 (2004).

[27] Nath, N. and Chilkoti, A., "A colorimetric gold nanoparticle sensor to interrogate biomolecular interactions in real time on a surface," Anal. Chem. 74, 504-509 (2002).

[28] Zeng, S., Yong, K.-T., Roy, I., Dinh, X.-Q., Yu, X., and Luan, F., "A review on functionalized gold nanoparticles for biosensing applications," Plasmonics 6(3), 491-506 (2011).

[29] Dahlin, A. B., Chen, S., Jonsson, M. P., Gunnarsson, L., Käll, M., and Höök, F., "High-resolution microspectroscopy of plasmonic nanostructures for miniaturized biosensing," Anal. Chem. 81, 6572-6580 (2009).

[30] Chen, S., Svedendahl, M., Antosiewicz, T. J., and Käll, M., "Plasmon-enhanced enzyme-linked immonosorbent assay on large arrays of individual particles made by electron beam lithography," ACS Nano 7, 8824-8832 (2013).

[31] Zijlstra, P., Paulo, P. M. R., and Orrit, M., "Optical detection of single non-absorbing molecules using the surface plasmon resonance of a gold nanorod," Nature Nanotechnol. 7, 379-382 (2012).

[32] Claudio, V., Dahlin, A. B., and Antosiewicz, T. J., "Single-particle plasmon sensing of discrete molecular events: Binding position versus signal variations for different sensor geometries," J. Phys. Chem. C 118, 6980-6988 (2014).

[33] Sannomiya, T., Hafner, C., and Voros, J., "In situ sensing of single binding events by localized surface plasmon resonance," Nano Lett. 8, 3450-2455 (2008).

[34] Unger, A., Rietzler, U., Berger, R., and Kreiter, M., "Sensitivity of crescent-shaped metal nanoparticles to attachment of dielectric colloids," Nano Lett. 9, 2311-2315 (2009).

[35] Ahijado-Guzmán, R., Prasad, J., Rosman, C., Henkel, A., Tome, L., Schneider, D., Rivas, G., and Sönnichsen, C., "Plasmonic nanosensors for simultaneous quantification of multiple protein-protein binding affinities," Nano Lett. 14, 5528-5532 (2014).

[36] Squires, T. M., Messinger, R. J., and Manalis, S. R., "Making it stick: Convection, reaction and diffusion in surface-based biosensors," Nat. Biotechnol. 26, 417-426 (2008). 
[37] Häfele, V., Trügler, A., Hohenester, U., Hohenau, A., Leitner, A., and Krenn, J. R., "Local refractive index sensitivity of gold nanodisks," Opt. Express 23, 10293-10300 (Apr 2015).

[38] Leitgeb, V., Trugler, A., Kostler, S., Krug, M. K., Hohenester, U., Hohenau, A., Leitner, A., and Krenn, J. R., "Three dimensional sensitivity characterization of plasmonic nanorods for refractometric biosensors," Nanoscale 8, 2974-2981 (2016).

[39] Salary, M. M. and Mosallaei, H., "A quasi-static continuum model describing interactions between plasmons and non-absorbing biomolecules," J. Appl. Phys. 117, 234303 (2015).

[40] Lüthgens, E. and Janshoff, A., "Equilibrium coverage fluctuations: A new approach to quantify reversible adsorption of proteins," Chem. Phys. Chem. 6, 444-448 (2005).

[41] Jokić, I., Djurić, Z., Frantlović, M., Radulović, K., Krstajić, P., and Jokić, Z., "Fluctuations of the number of adsorbed molecules in biosensors due to stochastic adsorption-desorption processes coupled with mass transfer," Sens. Actuators B Chem. 166-167, 535-543 (2012). 